\title{
Comparison of Performance Predictions for New Low- Thrust Trajectory Tools
}

\author{
Tara Polsgrove ${ }^{*}$, Larry Kos ${ }^{\dagger}$ and Randall Hopkins ${ }^{\ddagger}$ \\ Marshall Space Flight Center, Huntsville, Alabama, 35812 \\ Tracie Crane ${ }^{8}$ \\ Qualis Corporation, Huntsville, Alabama, 35806
}

\begin{abstract}
Several low thrust trajectory optimization tools have been developed over the last $3 \frac{1}{2}$ years by the Low Thrust Trajectory Tools development team. This toolset includes both low-medium fidelity and high fidelity tools which allow the analyst to quickly research a wide mission trade space and perform advanced mission design. These tools were tested using a set of reference trajectories that exercised each tool's unique capabilities. This paper compares the performance predictions of the various tools against several of the reference trajectories. The intent is to verify agreement between the high fidelity tools and to quantify the performance prediction differences between tools of different fidelity levels.
\end{abstract}

\section{Nomenclature}

$b b=$ constant term in constant specific impulse thrust efficiency function

$C_{3}=$ two times the orbital energy or the square of $V_{\infty}$

$d d=$ proportional term in thrust efficiency function, sec

$\eta \quad=$ propulsion system efficiency

$I_{s p} \quad=$ specific impulse

$m_{p s} \quad=$ mass of the propulsion system

$m_{t s} \quad=$ mass of the thrust subsystem

$m_{w} \quad=$ mass of the power subsystem

$P_{0} \quad=$ total power

$P_{\text {in }} \quad=$ power input to the propulsion system $\left(P_{0}-P_{s c}\right)$

$P_{j} \quad=$ jet power

$P_{s c} \quad=$ power used for spacecraft functions other than propulsion

$V_{\infty} \quad=$ planet relative hyperbolic excess velocity on outgoing or incoming asymptote

\section{Introduction}

$\mathrm{D}$ URING the summer of 2002 the National Aeronautics and Space Administration began the task of renovating its low thrust trajectory analysis capability. The tools at the time were difficult to master, limited in capabilities, and not uniform across field centers. An inter-center team of experts was established, the Low-Thrust Trajectory Tools team or LTTT, to correct the situation. This $3 \frac{1 / 2}{2}$ year project has resulted in the most capable tools for low thrust trajectory optimization ever demonstrated. ${ }^{1}$

During this development process a set of 32 reference missions were identified that would be relevant to future NASA science needs and provide each of the 5 new LTTT tools the opportunity to demonstrate its range of capabilities. Ongoing tool beta testing is focused on completing all applicable reference missions for each of the LTTT tools. A subset of these reference missions will be discussed in this paper. Results from the newly developed LTTT tools, and some predecessor tools will be compared. Comparison discussions in this paper are high level and do not address differences in numerical integration techniques, ephemeris data, or optimization schemes. While

\footnotetext{
*Aerospace Engineer, Advanced Concepts Office, NASA MSFC Mail Code VP11.

${ }^{\dagger}$ Aerospace En gineer, Advanced Concepts Office, NASA MSFC Mail Code VP11, AIAA Senior Member.

‡ Aerospace Engineer, Advanced Concepts Office, NASA MSFC Mail Code VP11.

${ }^{\S}$ Engineer, Advanced Concepts Office, NASA MSFC Mail Code VP11.
} 
comparing these new tools to each other and to predecessor tools is useful in building confidence and should illuminate the varying capabilities of the tools, the beta testing effort is not a tool validation. The tools can only be validated with flight demonstrations or by comparing against flight qualified software. It is hoped that one of the LTTT tools will complete the process to become flight qualified, and can then be used to validate the remainder of the LTTT suite.

A brief discussion of the tools, the reference missions and a subset of the results will be provided in this paper along with a comparison of the performance predictions.

\section{The Tools}

The following is a brief description of the tools that will be compared in this paper. Each of the tools described below are assigned relative fidelity levels of low, mediu $\mathrm{m}$, and high. The first four, CHEBYTOP and VARITOP, SEPTOP, and SAIL were not part of the LTTT development effort. Prior to the LTTT activity, these 4 tools were the primary low-thrust trajectory analysis tools for many of NASA's preliminary design studies. The remaining 5 tools were released earlier this year at the conclusion of the LTTT task. The LTTT development effort has yielded higher fidelity tools that are extensively documented and easier for trajectory analysts to use. Results from almost all of these tools are presented in section IV. Due to budget cuts, beta testing results from SNAP are not yet available, however its description is included to complete the introduction of the LTTT suite of tools .

\section{A. CHEBYTOP}

The pseudo-acronym stands for Chebychev Trajectory Optimization Program. This tool was originally written by Forrester Johnson \& colleagues at The Boeing Company ${ }^{2-5}$, and later modified by Carl Sauer at the Jet Propulsion Laboratory (JPL), and analysts at Glenn Research Center (GRC). CHEBYTOP uses Chebychev polynomials to represent state variables. These polynomials are then differentiated and integrated in closed form to solve a variable-thrust trajectory. This solution can then be used to approximate the performance of the constant thrust trajectory. CHEBYTOP is not capable of analyzing multileg missions such as round trip flights, or intermediate flybys. It is also limited to interplanetary missions with only the Sun's gravity field. For that reason it will not be used on reference missions requiring this type of analysis. CHEBYTOP is considered a low-fidelity program compared to other tools in this development effort, but it is highly valued for its capability to rapidly assess large trade spaces.

\section{B. VARITOP/SEPTOP/SAIL}

The names represent the Variational calculus Trajectory Optimization Program, the Solar Electric Propulsion Trajectory Optimization Program, and the solar Sail program. VARITOP is the most general of the suite, handling NEP (nuclear electric propulsion) as well as SEP (solar electric propulsion) and sail trajectories, however SEP and sail trajectories are more accurately represented in the SEPTOP and SAIL programs respectively. These three tools were created by Carl Sauer at JPL, and are based on the same mathematical formulation sharing many common subroutines. The calculus of variations is used in the formulation of state and co-state equations which are integrated numerically to solve a two-point boundary value problem. Optimization utilizes transversality conditions associated with the variational calculus, primer vector theory, and Pontryagin's maximum principle. ${ }^{6}$

SEPTOP can simulate thruster throttling and staging, and was used to provide trajectory support for the Deep Space 1 mission, DS1. During the DS1 mission analysis, an n-body option (the ability to operate in multiple gravitational fields) was added to the code, but the implementation prevented its use during close passes of planetary bodies (which was acceptable for the DS1 mission.) With the exception of the limited n-body analysis capability, these programs are two-body, sun-centered tools and are not able to analyze planet-centered trajectories beyond a simple escape or capture maneuver. For this reason they will not be used to analyze some of the reference missions requiring moon tours at the destination planet. These tools are of a higher fidelity than CHEBYTOP, and for the purposes of this paper will be considered medium fidelity tools.

\section{MALTO}

The Mission Analysis Low-Thrust Optimization tool, MALTO, was developed at JPL based on an approach described by Sims and Flanagan. ${ }^{7-9}$ Additional algorithmic testing was done with the tool GALLOP (Gravity-Assist Low-thrust Local Optimization Program) ${ }^{10}$ a joint project between JPL and Purdue University. MALTO uses many impulsive burns to simulate a continuous burn trajectory about a single gravitational source. The trajectory path and various parameters can be plotted in the graphical user interface or GUI. The thruster and power system modeling is 
compatible with the VARITOP suite of programs. Optimization is carried out by the SNOPT code developed by Dr. Philip Gill at the University of California San Diego. ${ }^{11}$ MALTO is considered a mid-fidelity tool.

\section{Copernicus}

Copernicus was developed by the University of Texas at Austin under technical direction from the Johnson Space Center. ${ }^{12-14}$ Copernicus is a generalized trajectory design and optimization program that allows the user to model from simple to complex missions using many objective functions, optimization variables, and constraint options. By selecting from a variety of integration and optimization methods and setting tolerances, the user can dial in the analysis fidelity level Using the same framework one can design a simple impulsive maneuver about a point mass or model multiple spacecraft in complex gravitational fields performing optimized finite burns with multiple or hybrid propulsion systems. The ability to model multiple spacecraft is helpful for rendezvous and targeting problems. In the low thrust regime, Copernicus can optimize both constant specific impulse and variable specific impulse trajectories. The trajectory path can be plotted in the GUI during the optimization process, so the user has real time feedback on the progress of the optimization. Copernicus is an $n$-body tool and is considered high fidelity.

\section{E. Mystic}

Mystic was developed by Greg Whiffen and others at JPL ${ }^{15-19}$ It uses a Static/Dynamic optimal Control method, SDC, to perform nonlinear optimization. It is an n-body tool and can analyze interplanetary missions as well as planet-centered missions in complex gravity fields. One of Mystic's strengths is the ability to automatically find and use gravity assists when they are beneficial. Mystic also allows the user to plan for various spacecraft operation and navigation activities during the mission. The trajectory path and various parameters can be plotted in the GUI. Mystic is a high fidelity optimization and simulation progra $\mathrm{m}$

\section{F. OTIS 4.0}

The Optimal Trajectories by Implicit Simulation program, OTIS 4, was developed by GRC \& Boeing. OTIS 3 is often thought of as primarily a launch vehicle trajectory and analysis program, but it has had a fairly robust interplanetary capability. With a mathematical re-formulation ${ }^{20-22}$ the tool is now more capable of performing very accurate interplanetary mission analyses, including low-thrust trajectories. The tool is named for its original implicit integration method, but also includes capabilities for explicit integration and analytic propagation. Vehicle models can be very sophisticated, and can be simulated through six degrees of freedom. OTIS uses SLSQP ${ }^{23}$ and SNOPT ${ }^{11}$ to solve the underlying nonlinear progra mming problem associated with the solution of the implicit integration method. This tool is a high fidelity optimization and simulation program. It is best used to optimize interactions between power, propulsion, and other system models.

\section{G. SNAP}

Spacecraft N-body Analysis Program, SNAP, was developed at GRC with help from Mike Martini of Analex Corporation. $^{24}$ It is a high fidelity trajectory propagation program that can be used for planet-centered and some interplanetary analysis. Many of SNAP's high fidelity features are especially useful for planet-centered trajectories, such as atmospheric drag, shadowing, and high order gravity models. Solar radiation pressure can also be modeled. SNAP uses a Runge-Kutta Fehlberg method of order 7-8 to propagate trajectories. SNAP is different from the other tools because it is planet-centered, and does not contain an optimizer.

\section{The Reference Missions}

A set of 32 mission categories was compiled during this activity and are given in Fig. 1. Tools are grouped by low, medium (or mid), and high fidelity levels, and into an E-C, or Earth-centered, category. Highlighted tools are part of the LTTT suite. Notice the limitation of the low fidelity tool CHEBYTOP. Many missions can not be analyzed with CHEBYTOP and are marked "n/a," or not applicable. In contrast, the high fidelity tools Copernicus, Mystic, and OTIS can perform almost all of the reference missions. There are a total of 191 possible cases identified in this figure, not including the sub-cases under many of the main reference missions.

Missions 1, 16, 17, 26, 28 and selected sub-cases will be discussed in this paper. These missions were chosen both to illustrate comparisons between the tools and to demonstrate the wide range of applications for these tools. The results for the remainder of the completed reference missions can be found on the LTTT website (http://www.inspacepropulsion.com/LTTT/). Instructions and guidelines for using the website are given in a 
companion paper by Larry Kos, "Overview of the Development of a Suite of Low-Thrust Trajectory Analysis Tools."

\begin{tabular}{|c|c|c|c|c|c|c|c|c|c|c|}
\hline \multirow[b]{2}{*}{ Tool: } & Low & \multicolumn{2}{|c|}{$\mathrm{E}-\mathrm{C} \mathrm{E}-\mathrm{C}$} & \multicolumn{3}{|c|}{ mid mid mid } & \multirow[b]{2}{*}{$\frac{\text { mid }}{\stackrel{\circ}{\frac{5}{\Sigma}}}$} & \multicolumn{3}{|c|}{ high high high } \\
\hline & 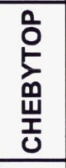 & 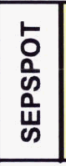 & $\sum_{\infty}^{0}$ & 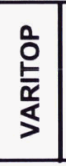 & $\begin{array}{l}\text { 음 } \\
\text { 品 }\end{array}$ & $\overline{\bar{心}}$ & & 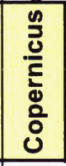 & $\sum_{\Sigma}^{0}$ & $\frac{n}{b}$ \\
\hline 1) Earth - Mars flyby & $\checkmark$ & $\mathrm{n} / \mathrm{a}$ & $\checkmark$ & $\checkmark$ & $\checkmark$ & $\checkmark$ & $\checkmark$ & $\checkmark$ & $\checkmark$ & $\checkmark$ \\
\hline 2) Earth - Mars rendezvous & $\checkmark$ & $\mathrm{n} / \mathrm{a}$ & $\mathrm{n} / \mathrm{a}$ & $\checkmark$ & $\checkmark$ & $\checkmark$ & $\checkmark$ & $\checkmark$ & $\checkmark$ & $\checkmark$ \\
\hline 3) Earth - Mars flyby - Vesta $\left(7^{\circ}\right)$ flyby & $\mathrm{n} / \mathrm{a}$ & $\mathrm{n} / \mathrm{a}$ & $\mathrm{n} / \mathrm{a}$ & $\checkmark$ & $\checkmark$ & $\checkmark$ & $\checkmark$ & $\checkmark$ & $\checkmark$ & $\checkmark$ \\
\hline 4) Earth - Mars flyby - Vesta rendezvous & $\mathrm{n} / \mathrm{a}$ & $\mathrm{n} / \mathrm{a}$ & $\mathrm{n} / \mathrm{a}$ & $\checkmark$ & $\checkmark$ & $\checkmark$ & $\checkmark$ & $\checkmark$ & $\checkmark$ & $\checkmark$ \\
\hline 5) Earth - Jupiter flyby & $\checkmark$ & $\mathrm{n} / \mathrm{a}$ & $\checkmark$ & $\checkmark$ & $\checkmark$ & $\checkmark$ & $\checkmark$ & $\checkmark$ & $\checkmark$ & $\checkmark$ \\
\hline 6) Earth - Venus flyby - Jupiter flyby & $\mathrm{n} / \mathrm{a}$ & $\mathrm{n} / \mathrm{a}$ & $\mathrm{n} / \mathrm{a}$ & $\checkmark$ & $\checkmark$ & $\checkmark$ & $\checkmark$ & $\checkmark$ & $\checkmark$ & $\checkmark$ \\
\hline 7) Earth - Tempel 1 Rendezvous & $\checkmark$ & $\mathrm{n} / \mathrm{a}$ & $\mathrm{n} / \mathrm{a}$ & $\checkmark$ & $\checkmark$ & $\mathrm{n} / \mathrm{a}$ & $\checkmark$ & $\checkmark$ & $\checkmark$ & $\checkmark$ \\
\hline 8) Earth - Venus/Vns/Jupiter flybys - Pluto flyby & $\mathrm{n} / \mathrm{a}$ & $\mathrm{n} / \mathrm{a}$ & $\mathrm{n} / \mathrm{a}$ & $\checkmark$ & $\checkmark$ & $\checkmark$ & $\checkmark$ & $\checkmark$ & $\checkmark$ & $\checkmark$ \\
\hline 9) Earth - [>1 rev around the Sun] - Jupiter flyb] & $\checkmark$ & $\mathrm{n} / \mathrm{a}$ & $\checkmark$ & $\checkmark$ & $\checkmark$ & $\checkmark$ & $\checkmark$ & $\checkmark$ & $\checkmark$ & $\checkmark$ \\
\hline 10) Earth - Venus flyby - Mercury $\left(7^{\circ}\right)$ rendezvou & $\mathrm{n} / \mathrm{a}$ & $\mathrm{n} / \mathrm{a}$ & $\mathrm{n} / \mathrm{a}$ & $\checkmark$ & $\checkmark$ & $\checkmark$ & $\checkmark$ & $\checkmark$ & $\checkmark$ & $\checkmark$ \\
\hline 11) Earth - Tempel 1 Rendezvous - Earth Flyby & $\mathrm{n} / \mathrm{a}$ & $\mathrm{n} / \mathrm{a}$ & $\mathrm{n} / \mathrm{a}$ & $\checkmark$ & $\checkmark$ & $\mathrm{n} / \mathrm{a}$ & $\checkmark$ & $\checkmark$ & $\checkmark$ & $\checkmark$ \\
\hline 12) Mars Sample Return & $\mathrm{n} / \mathrm{a}$ & $\mathrm{n} / \mathrm{a}$ & $\mathrm{n} / \mathrm{a}$ & $\checkmark$ & $\checkmark$ & $\mathrm{n} / \mathrm{a}$ & $\checkmark$ & $\checkmark$ & $\checkmark$ & $\checkmark$ \\
\hline 13) Classic minimum time to Mars, circ/coplanan & $\checkmark$ & $\mathrm{n} / \mathrm{a}$ & $\checkmark$ & $\checkmark$ & $\checkmark$ & $\checkmark$ & $\checkmark$ & $\checkmark$ & $\checkmark$ & $\checkmark$ \\
\hline 14) Comet Sample Return & $\mathrm{n} / \mathrm{a}$ & $\mathrm{n} / \mathrm{a}$ & $\mathrm{n} / \mathrm{a}$ & $\checkmark$ & $\checkmark$ & $\mathrm{n} / \mathrm{a}$ & $\checkmark$ & $\checkmark$ & $\checkmark$ & $\checkmark$ \\
\hline 15) Multiple Asteroid Rendezvous & $\mathrm{n} / \mathrm{a}$ & $\mathrm{n} / \mathrm{a}$ & $\mathrm{n} / \mathrm{a}$ & $\checkmark$ & $\checkmark$ & $\checkmark$ & $\checkmark$ & $\checkmark$ & $\checkmark$ & $\checkmark$ \\
\hline 16) $0.5 \mathrm{AU}$ polar (incl. $45^{\circ}$ to the ecliptic) orbiter & $\mathrm{n} / \mathrm{a}$ & $\mathrm{n} / \mathrm{a}$ & $\checkmark$ & $\checkmark$ & $\checkmark$ & $\checkmark$ & $\checkmark$ & $\checkmark$ & $\checkmark$ & $\checkmark$ \\
\hline 17) 5-years to Jupiter/Europa Orbiter & $\checkmark$ & $\mathrm{n} / \mathrm{a}$ & $\mathrm{n} / \mathrm{a}$ & $\checkmark$ & $\checkmark$ & $\mathrm{n} / \mathrm{a}$ & $\checkmark$ & $\checkmark$ & $\checkmark$ & $\checkmark$ \\
\hline 18) 8-years to Saturn/Titan Orbiter & $\checkmark$ & $\mathrm{n} / \mathrm{a}$ & $\mathrm{n} / \mathrm{a}$ & $\checkmark$ & $\checkmark$ & $\mathrm{n} / \mathrm{a}$ & $\checkmark$ & $\checkmark$ & $\checkmark$ & $\checkmark$ \\
\hline 19) 10-years to Uranus/Titania Orbiter & $\checkmark$ & $\mathrm{n} / \mathrm{a}$ & $\mathrm{n} / \mathrm{a}$ & $\checkmark$ & $\checkmark$ & $\mathrm{n} / \mathrm{a}$ & $\checkmark$ & $\checkmark$ & $\checkmark$ & $\checkmark$ \\
\hline 20) 12-years to Neptune/Triton Orbiter & $\checkmark$ & $\mathrm{n} / \mathrm{a}$ & $\mathrm{n} / \mathrm{a}$ & $\checkmark$ & $\checkmark$ & $\mathrm{n} / \mathrm{a}$ & $\checkmark$ & $\checkmark$ & $\checkmark$ & $\checkmark$ \\
\hline 21) 12-years to Pluto/Charon Orbiter & $\checkmark$ & $\mathrm{n} / \mathrm{a}$ & $\mathrm{n} / \mathrm{a}$ & $\checkmark$ & $\checkmark$ & $\mathrm{n} / \mathrm{a}$ & $\checkmark$ & $\checkmark$ & $\checkmark$ & $\checkmark$ \\
\hline 22) 6-years to Jupiter (Moon) Tour & $\mathrm{n} / \mathrm{a}$ & $\mathrm{n} / \mathrm{a}$ & $\mathrm{n} / \mathrm{a}$ & $\mathrm{n} / \mathrm{a}$ & $\mathrm{n} / \mathrm{a}$ & $\mathrm{n} / \mathrm{a}$ & $\mathrm{n} / \mathrm{a}$ & $\checkmark$ & $\checkmark$ & $\checkmark$ \\
\hline 23) 9-years to Saturn (Moon) Tour & $\mathrm{n} / \mathrm{a}$ & $\mathrm{n} / \mathrm{a}$ & $\mathrm{n} / \mathrm{a}$ & $\mathrm{n} / \mathrm{a}$ & $\mathrm{n} / \mathrm{a}$ & $\mathrm{n} / \mathrm{a}$ & $\mathrm{n} / \mathrm{a}$ & $\checkmark$ & $\checkmark$ & $\checkmark$ \\
\hline 24) 11-years to Uranus (Moon) Tour & $\mathrm{n} / \mathrm{a}$ & $\mathrm{n} / \mathrm{a}$ & $\mathrm{n} / \mathrm{a}$ & $\mathrm{n} / \mathrm{a}$ & $\mathrm{n} / \mathrm{a}$ & $\mathrm{n} / \mathrm{a}$ & $\mathrm{n} / \mathrm{a}$ & $\checkmark$ & $\checkmark$ & $\checkmark$ \\
\hline 25) 13-years to Neptune (Moon) Tour & $\mathrm{n} / \mathrm{a}$ & $\mathrm{n} / \mathrm{a}$ & $\mathrm{n} / \mathrm{a}$ & $\mathrm{n} / \mathrm{a}$ & $\mathrm{n} / \mathrm{a}$ & $\mathrm{n} / \mathrm{a}$ & $\mathrm{n} / \mathrm{a}$ & $\checkmark$ & $\checkmark$ & $\checkmark$ \\
\hline 26) 12-years to Pluto Tour & $\mathrm{n} / \mathrm{a}$ & $\mathrm{n} / \mathrm{a}$ & $\mathrm{n} / \mathrm{a}$ & $\mathrm{n} / \mathrm{a}$ & $\mathrm{n} / \mathrm{a}$ & $\mathrm{n} / \mathrm{a}$ & $\mathrm{n} / \mathrm{a}$ & $\checkmark$ & $\checkmark$ & $\checkmark$ \\
\hline 27) Kuiper Belt-Pluto Explorer & $\mathrm{n} / \mathrm{a}$ & $\mathrm{n} / \mathrm{a}$ & $\mathrm{n} / \mathrm{a}$ & $\checkmark$ & $\checkmark$ & $\checkmark$ & $\checkmark$ & $\checkmark$ & $\checkmark$ & $\checkmark$ \\
\hline 28) Earth-Moon low thrust & $\mathrm{n} / \mathrm{a}$ & $\checkmark$ & $\checkmark$ & $\mathrm{n} / \mathrm{a}$ & $\mathrm{n} / \mathrm{a}$ & $\mathrm{n} / \mathrm{a}$ & $\mathrm{n} / \mathrm{a}$ & $\checkmark$ & $\checkmark$ & $\checkmark$ \\
\hline 29) Earth Solar libration point mission(s) & $\mathrm{n} / \mathrm{a}$ & $\mathrm{n} / \mathrm{a}$ & $\checkmark$ & $\mathrm{n} / \mathrm{a}$ & $\mathrm{n} / \mathrm{a}$ & $\mathrm{n} / \mathrm{a}$ & $\mathrm{n} / \mathrm{a}$ & $\checkmark$ & $\checkmark$ & $\checkmark$ \\
\hline 30) $M W$ to GW interplanetary mission(s) & $\mathrm{n} / \mathrm{a}$ & $\mathrm{n} / \mathrm{a}$ & $\mathrm{n} / \mathrm{a}$ & $\checkmark$ & $\checkmark$ & $\mathrm{n} / \mathrm{a}$ & $\checkmark$ & $\checkmark$ & $\checkmark$ & $\checkmark$ \\
\hline 31) Earth/Sun/Moon 4-body/other "n-body" miss & $\mathrm{n} / \mathrm{a}$ & $\mathrm{n} / \mathrm{a}$ & $\checkmark$ & $\mathrm{n} / \mathrm{a}$ & $\mathrm{n} / \mathrm{a}$ & $\mathrm{n} / \mathrm{a}$ & $\mathrm{n} / \mathrm{a}$ & $\checkmark$ & $\checkmark$ & $\checkmark$ \\
\hline 32) Non-Keplerian/Other Orbits & $\mathrm{n} / \mathrm{a}$ & $\mathrm{n} / \mathrm{a}$ & $\checkmark$ & $\mathrm{n} / \mathrm{a}$ & $\mathrm{n} / \mathrm{a}$ & $\checkmark$ & $\checkmark$ & $\mathrm{n} / \mathrm{a}$ & $\mathrm{n} / \mathrm{a}$ & $\mathrm{n} / \mathrm{a}$ \\
\hline
\end{tabular}

Figure 1. Reference mission list and applicable analysis tools.

\section{A. Reference Mission 1: Earth - Mars Flyby}

A simple Earth to Mars flyby mission was selected as the first reference mission. This mission is performed using SEP. Solar array and engine models are specified in Figs. 2 and 3 respectively. Many of the mission parameters are fixed quantities and not part of the trajectory optimization. Mission constraints and objective function are as follows:

- Launch fro m Earth on Julian date 2452779.5 (May 20, 2003) -- fixed

- Arrive at Mars on Julian date 2452979.5 (Dec. 6, 2003) -- fixed

- Initial mass: $585 \mathrm{~kg}$ (fixed)

- Launch $V_{\infty}$ magnitude: $1.66 \mathrm{~km} / \mathrm{s}$ (fixed)

- Launch $V_{\infty}$ direction is free

- Propulsion Model: Electric Engine G (1 Engine)

- Power Model: apsa 1 solar array, $\mathrm{P} 0=6.0 \mathrm{~kW}, \mathrm{Psc}=0$ 
- Objective function: final mass

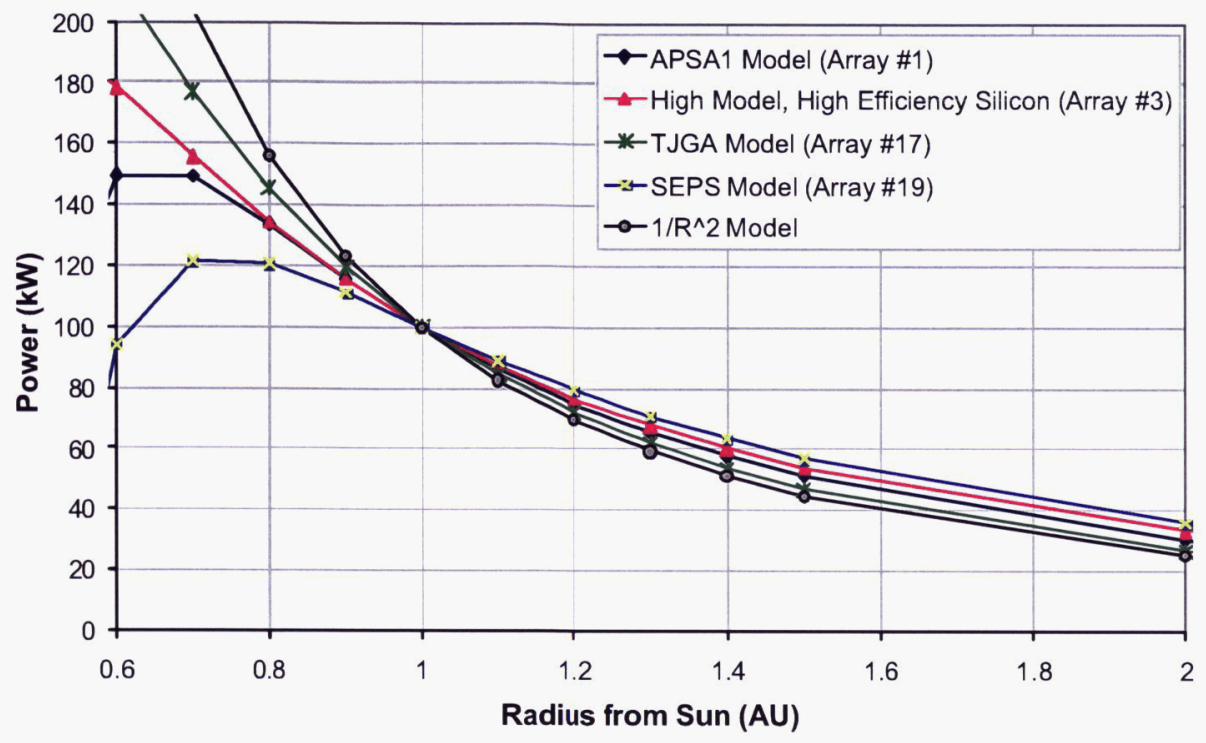

Figure 2. Solar array models, output power vs. radius from the Sun, no degradation over time

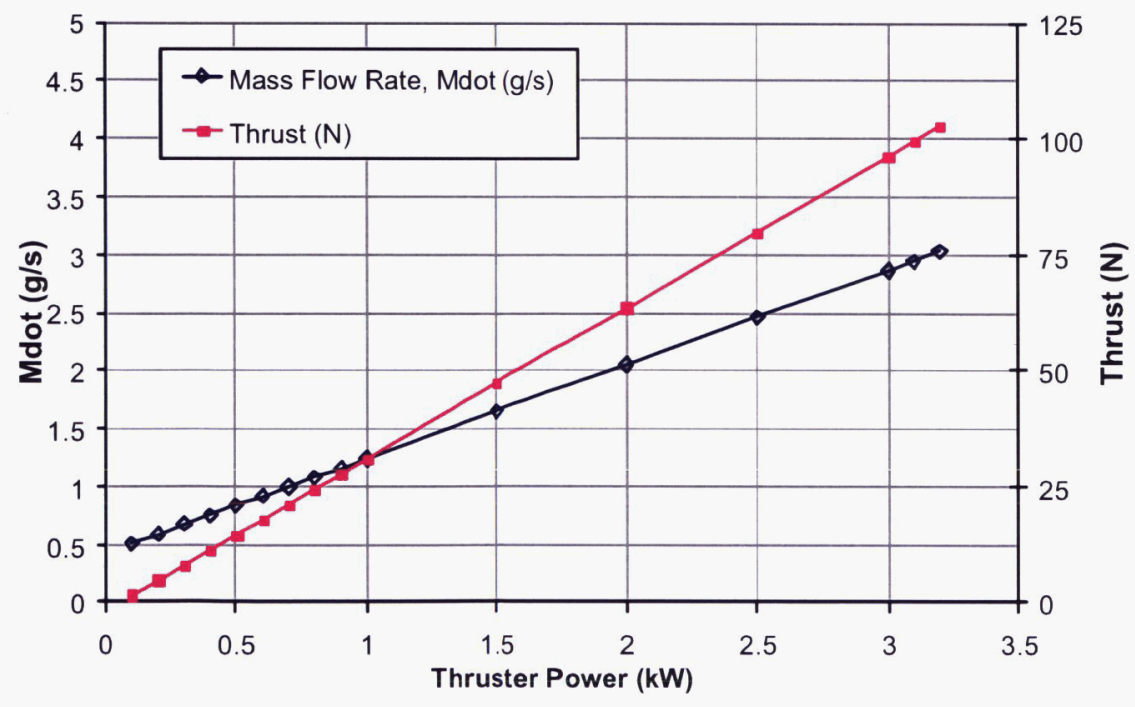

Figure 3. Propulsion model electric engine G (1 engine).

\section{B. Reference Mission 2f: Earth - Mars Rendezvous (Solar Sail)}

In almost all cases, each of the LTTT reference missions has a set of sub cases where constraints are relaxed or perturbed. Reference Mission 2 is nominally an SEP mission, but sub case f uses solar sail propulsion to travel from Earth escape and rendezvous with Mars. Mission constraints and objective function are as follows:

- launch date is free but around 2009

- Launch $V_{\infty}$ magnitude: $1.00 \mathrm{~km} / \mathrm{s}$ (fixed) 
- Launch $V_{\infty}$ direction is free

- $100 \times 100 \mathrm{~m}^{2}$ solar sail with a mass of $260.75 \mathrm{~kg}$.

- Objective function: minimize flight time

\section{Reference Mission 16: $0.5 \mathrm{AU} \times 4^{\circ}$ inclination heliocentric orbiter}

This reference mission uses a launch vehicle model and solar array model which are given in Figs. 4 and 2 respectively. Equation 1 defines the method for modeling thruster efficiency. Mission constraints and objective function are as follows:

- Earth Departure Conditions, January 1,2000:

- Delta III Launch Vehicle (launch vehicle model number 50)

○ $V_{\infty}=1.95 \mathrm{~km} / \mathrm{s}$ (optimized)

- Launch vehicle adapter mass ( $\mathrm{kadp})$ as a fraction of initial mass is 0.05

- Or, fix initial mass at $2204 \mathrm{~kg}$ and use $V_{\infty}$ above

- Target orbit:

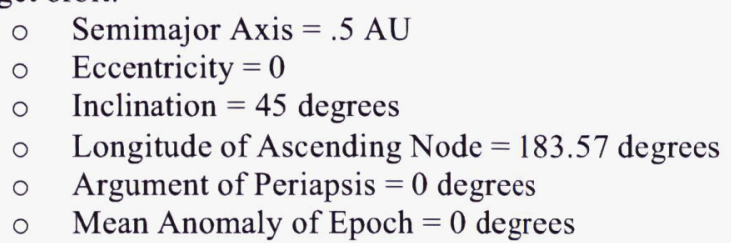

- Circular coplanar ephemeris

- Solar array model = "high"

- Propulsion System efficiency

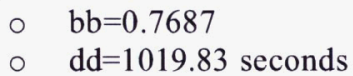

$$
\eta=\frac{P j}{P i n}=\frac{b b}{1+\left(\frac{d d}{I s p}\right)^{2}}=\frac{b b \times I s p^{2}}{I s p^{2}+d d^{2}}
$$

- $\quad$ Specific Impulse constant at 5316.4 seconds (optimized)

- Tankage Fraction kt is 0.1 , fraction of propellant mass

- Propulsion \& Power System Mass $30 \mathrm{~kg} / \mathrm{kW}$

- Power at $1 \mathrm{AU}$ is $40 \mathrm{~kW}$

- Maximum allowed power is $50 \mathrm{~kW}$

- $\quad$ Spacecraft Housekeeping Power $=0.25 \mathrm{~kW}$

- Flight Time is 630.6 days (optimized) 


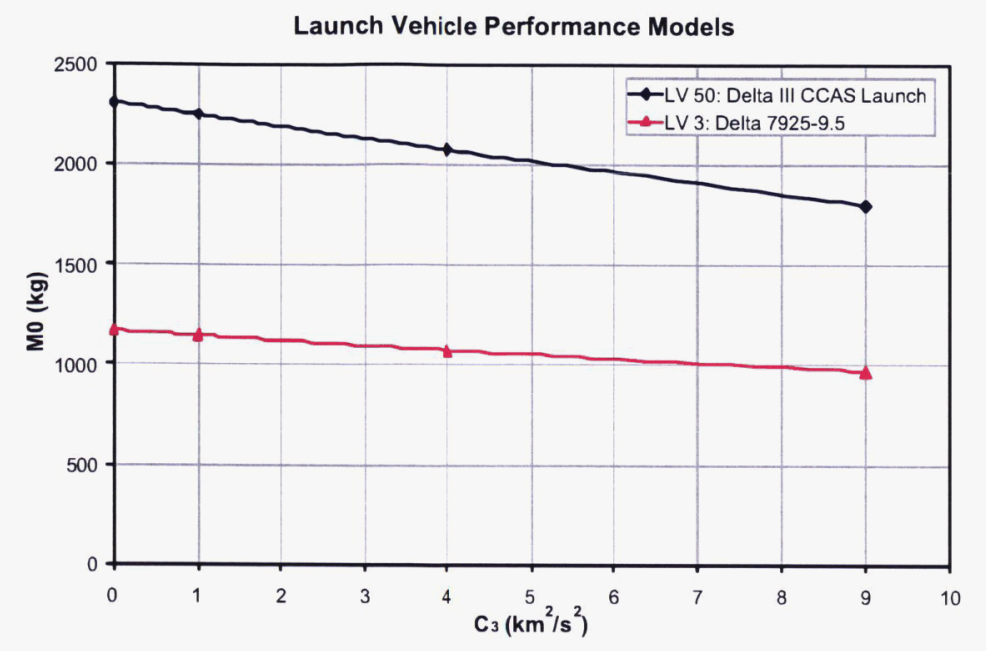

Figure 4. Launch vehicle performance (launch vehicle models 3, and 50).

\section{Reference Mission 16c: Solar Polar Rendezvous}

This variant on reference mission 16 requires the spacecraft to target a particular point on an orbit that is at a 90 degree inclination to the Sun, near circular, and at the Earth's semimajor axis. Launch vehicle and solar array performance curves are given in Figs. 4 and 2 respectively. Mission constraints and objective function are as follows:

- $\quad$ Launch from Earth on Julian date 2451452.5 (Oct. 1, 1999) -- fixed

- Rendezvous with Target orbit on Julian Date 2452852.5 (Aug. 1, 2003)

- Target Orbit:

- Semimajor Axis $=1.0 \mathrm{AU}$

- Eccentricity $=0.98$

- Inclination $=90$ degrees

- Right Ascension of Ascending Node $=10$ degrees

- Argument of Periapsis $=0$ degrees

- Mean Anomaly $=345$ degrees

- $\quad$ Launch Vehicle - Delta 7925-9.5; $10 \%$ contingency

- Launch $V_{\infty}$ magnitude, direction: free

- Propulsion Model: NSTAR Engine G (up to 4 Engines)

- Power Model: SEPS solar array, P0 = free,

- Objective function: final mass

\section{E. Reference Mission 17: 5-years to Jupiter/Europa Orbiter}

Mission number 17 is a mission to Jupiter's moon Europa. The spacecraft does not actually rendezvous with Europa in the trajectories presented in this paper, but shares Europa's orbit of Jupiter. Unlike the previous missions discussed, this NEP spacecraft begins its mission in Earth orbit and escapes on a spiral trajectory. Capture at Jupiter and descent to Europa's semimajor axis about Jupiter is also a spiraling trajectory. Mission constraints and objective function are as follows:

- Earth departure orbit is circular at an altitude of $1,000 \mathrm{~km}$

- Spiral escape to $\mathrm{C}_{3}=0$

- Depart from Earth sphere of influence (SOI) on Julian date $2457306.5(10 / 11 / 2015)$ - fixed at optimal

- Arrive at Jupiter SOI on Julian date 2459132.75 (10/10/2020) -- fixed

- Spiral descent to circular Jupiter parking orbit at an altitude of $600,989 \mathrm{~km}$ ( $\sim$ Europa altitude)

- Initial mass: $4310 \mathrm{~kg}$ (fixed)

- Propulsion and power system specific mass $=50 \mathrm{~kg} / \mathrm{kW}$ 
- $\quad$ Tankage fraction $=0.1$, (Tank mass/propellant mass $)$

- Propulsion Model: simple electric engine,

- Isp 6400 seconds, (optimal $+/-100$ seconds)

- 0.65 efficiency

- Power Model: constant power (nuclear), optimized to $24.85 \mathrm{~kW}$

- Objective function: final mass

\section{F. Reference Mission 26: 12-years to Pluto Tour}

Since the heliocentric portion of this mission is covered in reference mission 21 , this reference mission focuses only on the Pluto vicinity. The mission begins in a low circular polar orbit around Charon, then spirals out away from Charon and ends in a low circular polar orbit around Pluto. Mission constraints and objective function are as follows:

- Start Date: Jan 1, 2027 (fixed)

- Flight Time: 44 days (fixed)

- Initial mass: 6,100 kg (fixed)

- Initial State around Charon:

- Semi-major axis $=685 \mathrm{~km}$

- Inclination $=90 \mathrm{deg}$

- Eccentricity $=0$

- Final State around Pluto:

○ Semi-major axis $=1350 \mathrm{~km}$

- Inclination $=90 \mathrm{deg}$

- Eccentricity $<0.1$

- Propellant Mass Constraint: $\mathrm{mp}<115 \mathrm{~kg}$

- Propulsion and power system mass (alpha) $=50 \mathrm{~kg} / \mathrm{kW}$

- $\quad$ Tankage fraction $=0.1$, (Tank mass/propellant mass)

- Propulsion Model: simple electric engine,

- Isp 6100 seconds, (optimal $+/-100$ seconds)

- 0.65 efficiency

- Power Model: constant power (nuclear), $84.05 \mathrm{~kW}$ (fixed)

- Objective function: final mass

\section{G. Reference Mission 28a: Low Earth Orbit - Low Lunar Orbit with low thrust}

This mission begins in a 100,000 km circular Earth orbit and targets an orbit of $100 \mathrm{~km}$ altitude above the Moon at a polar inclination. Mission constraints and objective function are as follows:

- Propulsion System SEP

- Launch Date 05/20/2003 (fixed)

- End Date 09/07/2003 (fixed)

- Flight Time 110 days (fixed)

- Initial Mass $2,500 \mathrm{~kg}$ (fixed)

- P0 $20 \mathrm{~kW}$, constant throughout mission

- Isp 6000 seconds

- Efficiency $70 \%$

- Duty Cycle $100 \%$

- Initial States (EME2000) - fixed

$$
\begin{aligned}
\circ & \mathrm{a}=100,000 \mathrm{~km} \\
\circ & \mathrm{e}=0 \\
\circ & \mathrm{i}=28.5 \mathrm{deg}
\end{aligned}
$$

- Final State (Moon Centered) - altitude $=100 \mathrm{~km}$, circular with inclination $=90$ degrees

- Objective function: final mass 


\section{H. Reference Mission 28c: Earth - Lunar L1 Halo Orbit}

This is a subcase of reference mission 28, discussed above. For this case an Earth-Moon L1 halo orbit is the final destination. Mission constraints and objective function are the same as was given for Reference Mission 28 with the following exceptions:

- $\quad$ Time To Halo-Orbit-Insertion (HOI): 82.5751 days

- No Thrusting after HOI

- Total Flight Time: 118 days (fixed)

- Halo Orbit Target:

$$
\begin{aligned}
& \text { - } A x=10,000 \mathrm{~km} \\
& \text { - } \mathrm{Ay}=30,000 \mathrm{~km} \\
& \text { - } \mathrm{Az}=10,000 \mathrm{~km}
\end{aligned}
$$

- Propellant Mass: $<25 \mathrm{~kg}$

\section{The Results}

In this section the results of each tool are compared for the 8 reference missions selected. The obvious question is "which tool is right?" The answer: none of the tools have completed the long verification and validation process required to become flight-support software, but Mystic is significantly further along in this process than the other tools. At a minimum the discussion that follows should help to illustrate the capabilities and limitations of each of the tools. Unfortunately SNAP missions were not completed in time to report in this document, but should eventually be available for missions 1, 16, and 28. Once complete the SNAP missions will be available on the LTTT website. Note that in all tables, boxed values are values that were constrained.

\section{A. Reference Mission 1: Earth - Mars Flyby}

This relatively simple interplanetary mission is depicted in Fig. 5. This mission was first optimized using the tool SEPTOP. All other tools are compared to that initial trajectory. Results from each of the tools tested are given in Table 1.

\section{CHEBYTOP RESULTS}

This solar-electric mission was originally analyzed in SEPTOP. Propulsion and power models were Electric Engine G (1 Engine) with APSA1 solar arrays, respectively. While CHEBYTOP has no capability to incorporate sophisticated array or engine models, it was able to duplicate this mission well. There were two key factors that allowed a close CHEBYTOP comparison. First, the solar array power and the thrust generation power were decoupled. During the thrusting portion of the SEPTOP mission, the input power ranged from $6 \mathrm{~kW}$ to $4.2 \mathrm{~kW}$, never falling below the max thruster power. This meant that from a thrust point of view the mission was run with a constant power trajectory that could be modeled as an NEP case. The second factor is a result of the first. Because the thruster operated at its max power during the entire thrusting phase, a single $I_{s p}$ and efficiency data point could be used in place of the more sophisticated thruster model.

The final mass is $0.05 \mathrm{~kg}$ of the SEPTOP mission, and the thrusting time is only 0.6 days off of the reported SEPTOP value.

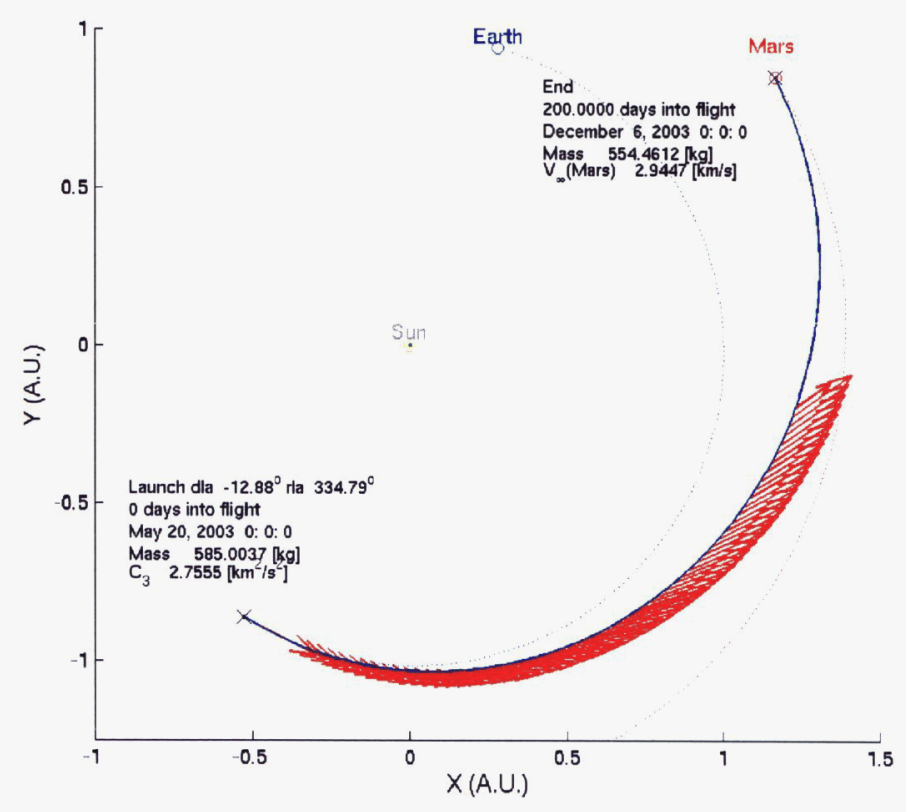

Figure 5. Reference mission 1 trajectory plot (Mystic). 
Table1. Reference mission 1 results.

\begin{tabular}{|c|c|c|c|c|c|c|c|}
\hline Parameter & Units & CHEBYTOP & SEPTOP & MALTO & Copernicus & Mystic & OTIS \\
\hline Departure Date & $\mathrm{n} / \mathrm{a}$ & $5 / 20 / 2003$ & $5 / 20 / 2003$ & $5 / 20 / 2003$ & $5 / 20 / 2003$ & $5 / 20 / 2003$ & $5 / 20 / 2003$ \\
\hline $\begin{array}{l}\text { Launch Vinf } \\
\text { Magnitude }\end{array}$ & $\mathrm{km} / \mathrm{s}$ & 1.66 & 1.66 & 1.66 & 1.66 & 1.66 & 1.66 \\
\hline $\begin{array}{c}\text { Heliocentric Flight } \\
\text { Time }\end{array}$ & days & 200 & 200 & 200 & 200 & 200 & 200 \\
\hline Initial Mass & $\mathrm{kg}$ & 585 & 585 & 585 & 585 & 585 & 585 \\
\hline Final Mass & $\mathrm{kg}$ & 554.4 & 554.35 & 554.33 & 559.22 & 554.46 & 554.3 \\
\hline $\begin{array}{c}\text { Heliocentric } \\
\text { Thrusting Time }\end{array}$ & days & 125.6 & 125 & 125.8 & 200 & 125 & 125.8 \\
\hline Solar Array & & NEP & apsa 1 model & apsa 1 model & $1 / \mathrm{R}^{2}$ & apsal model & NEP \\
\hline Power at $1 \mathrm{AU}$ & $\mathrm{kWe}$ & 2.6 & 6 & 6 & --- & 6 & 2.6 \\
\hline $\begin{array}{l}\text { Thruster max } \\
\text { input power }\end{array}$ & $\mathrm{kWe}$ & 2.6 & 2.6 & 2.6 & 2.6 & 2.6 & 2.6 \\
\hline Thruster & & $\mathrm{n} / \mathrm{a}$ & $\begin{array}{c}\text { Electric Engine G } \\
(1 \text { Engine }) \\
\end{array}$ & $\begin{array}{c}\text { Electric Engine G } \\
(1 \text { Engine })\end{array}$ & $\mathrm{n} / \mathrm{a}$ & $\begin{array}{c}\text { Electric Engine } \mathrm{G} \\
\text { (1 Engine) }\end{array}$ & $\mathrm{n} / \mathrm{a}$ \\
\hline Specific Impulse & sec & 3,337 & 3,337 & 3,337 & 3,337 & 3,337 & 3,337 \\
\hline Efficiency & n.d. & 0.58096 & 0.58096 & 0.58096 & 1 & 0.58096 & 0.58096 \\
\hline Initial Thrust & $\mathrm{N}$ & 0.0923 & 0.0923 & 0.0923 & 0.1589 & 0.0923 & 0.0923 \\
\hline
\end{tabular}

2. MALTO RESULTS

MALTO is able to model this trajectory using the same propulsion and power models that are available in SEPTOP. The resulting trajectory compares more closely with SEPTOP than the results from the low fidelity tool CHEBYTOP. The final mass is only $0.02 \mathrm{~kg}$ different from SEPTOP, and the time spent thrusting is approximately 1 day different. MALTO simulated the trajectory using 32 segments, with the match point located at 50 percent of the total flight time.

3. COPERNICUS RESULTS

Unlike the SEPTOP ase where oversized solar arrays meet thruster needs during the entire mission, in the Copernicus case thrust varies with distance from the Sun. The initial thrust is higher than in the other missions, but the thrusting time is 75 days longer. The final mass for the Copernicus case is higher than the SEPTOP mass, but still within $1 \%$. It would be interesting to model this case similar to CHEBYTOP and use NEP or constant power rather than an SEP power profile.

4. MYSTIC RESULTS

Mystic, like MALTO, is able to use the same solar array and engine models as SEPTOP, but perhaps due to its higher fidelity optimization it is able to provide a slightly higher final mass.

5. OTIS RESULTS

OTIS results are in agreement with the results of the other tools, with the exception of Copernicus which was run at a different thrust level.

\section{B. Reference Mission 2f: Earth - Mars Rendezvous (Solar Sail)}

At least one of the LTTT tools was required to have solar sail trajectory analysis capability. This capability was incorporated into the medium fidelity tool MALTO. The medium fidelity tool was chosen in part because there was a separate development effort concurrent to LTTT with the specific task of developing a tool to perform high fidelity modeling of solar sail missions. This effort resulted in the S5 tool. ${ }^{25-26}$ While incorporating mid-fidelity solar sail trajectory optimization capability into the LTTT tools was not a duplication of effort, altering one of the high fidelity 
tools to do this would have been. Now that both development efforts are complete developers (if given the time) could make the S5 tool and the LTTT high fidelity tools work together.

Reference mission $2 \mathrm{f}$, a solar sail mission from the Earth to a rendezvous with Mars is depicted in Fig. 6 . Results from MALTO and SAIL are given in Table 2. Again values that were constrained and not optimized are outlined.

Table2. Reference mission 2 f results.

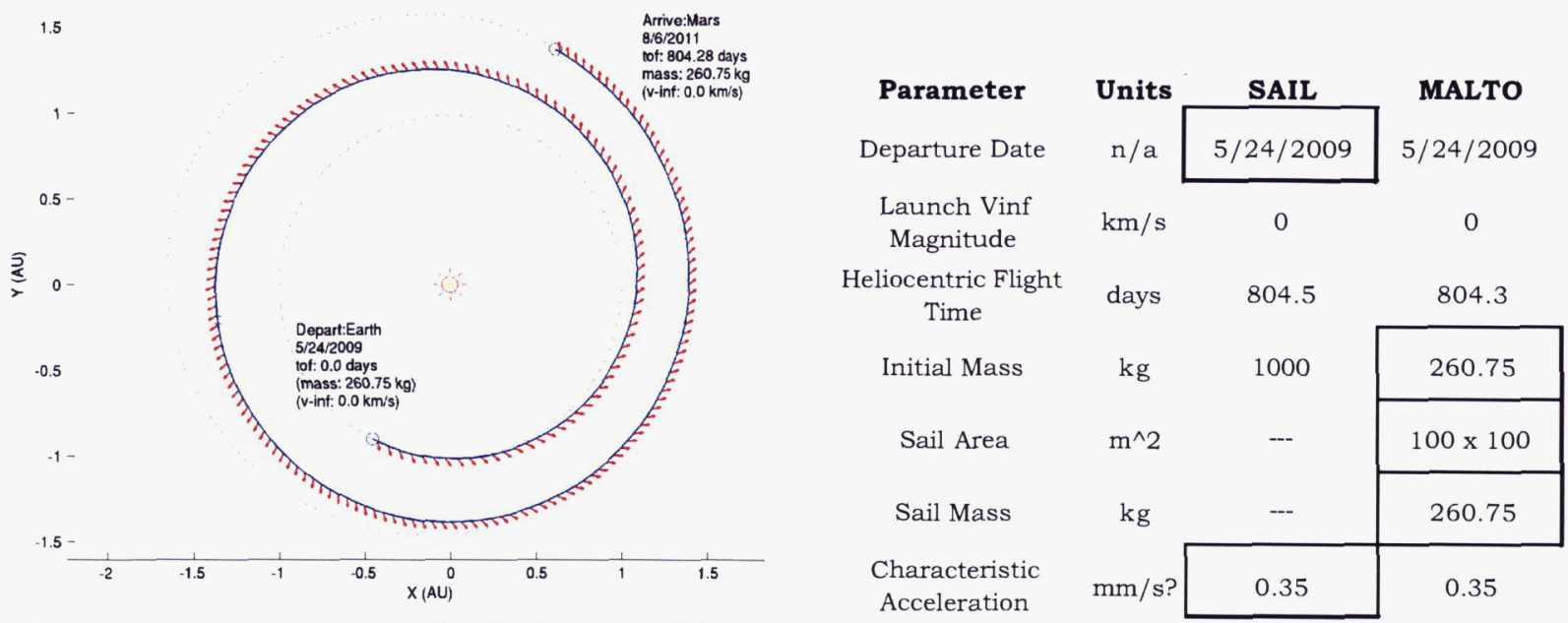

Figure 6. Reference mission $2 \mathrm{f}$ trajectory plot (MALTO).

\section{SAIL RESULTS}

In the SAIL program one can simply enter the characteristic acceleration rather than sail size and mass details. The thrust direction is optimized along the trajectory to minimize the flight time. The minimum flight time is 804.5 days.

2. MALTO RESULTS

MALTO uses sail area and mass inputs rather than characteristic acceleration. Sail area and mass numbers were chosen to yield the same characteristic acceleration as the SAIL case. MALTO reports a minimum flight time of 804.28 days.

\section{S5 RESULTS}

Results from the S5 tool are not given in Table 2 because they were not readily available to scrutinize, however sources indicate that the $\mathrm{S} 5$ tool predicts 808.2 days as a minimum time to perform this mission. This is a difference of less than $0.5 \%$ from the MALTO prediction.

\section{Reference Mission 16 \& 16c: 0.5 AU x $45^{\circ}$ inclination heliocentric orbiter \& Solar Polar ${ }^{\times 10}$ Rendezvous}

One of the goals in putting together the list of reference missions was to evaluate the LTTT tools in as many different types of solution spaces as possible. Reference missions 16 and $16 \mathrm{c}$ are high inclination trajectories in the inner solar system that require multiple revolutions around the sun to reach the destination orbit. Figure 7 illustrates one of the solutions for reference mission 16 . Figures 8 and 9 depict reference mission $16 \mathrm{c}$. These are challenging missions that cannot be analyzed with the low fidelity tool CHEBYTOP. Results from VARITOP,

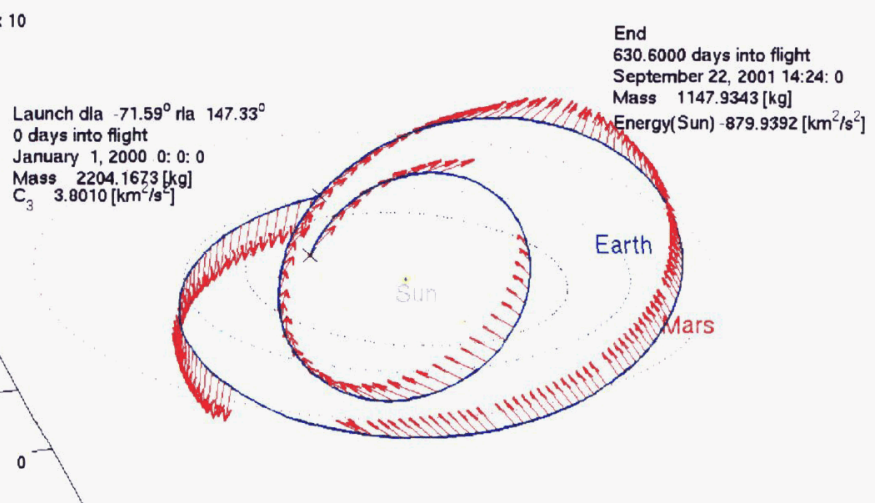
MALTO, Mystic, SEPTOP, and Copernicus are given in Table 3.

Figure 7. Reference mission 16 trajectory plot (Mystic).

11 
Table3. Reference mission $16 \& 16 \mathrm{c}$ results.

Reference Mission 16

\begin{tabular}{|c|c|c|c|c|}
\hline Parameter & Units & VARITOP & MALTO & Mystic \\
\hline Target Orbit & & $0.5 \mathrm{AUx} 45 \mathrm{deg}$ & $0.5 \mathrm{AUx} 45 \mathrm{deg}$ & $0.5 \mathrm{AUx} 45 \mathrm{deg}$ \\
\hline Departure Date & $\mathrm{n} / \mathrm{a}$ & $1 / 1 / 2000$ & $1 / 1 / 2000$ & $1 / 1 / 2000$ \\
\hline Launch Vehicle & & $\begin{array}{c}\text { Delta III (LV } \\
\# 50)\end{array}$ & $\begin{array}{c}\text { Delta III (LV } \\
\# 50)\end{array}$ & --- \\
\hline
\end{tabular}

Launch Vinf

Magnitude

Heliocentric Flight Time

Initial Mass

Final Mass

Heliocentric

Thrusting Time

Solar Array

Power at $1 \mathrm{AU}$

Max Power Limit

Thruster

Specific Impulse

Efficiency (bb)

Efficiency (dd)

Initial Thrust $\mathrm{km} / \mathrm{s} \quad 1.95$

days

630.6

2204

\begin{tabular}{|l|l|}
\hline \multicolumn{1}{|c|}{1.95} & 1.95 \\
\hline 630.6 & 630.6 \\
\hline
\end{tabular}

$\mathrm{kg}$

$1,121.52$

2204

2204

1138.21

1131.89

days

540.37

515.84

529.704

\begin{tabular}{|c|c|c|}
\hline "high" model & $1 / \mathrm{R}^{2}$ & $1 / \mathrm{R}^{2}$ \\
\hline 40 & 40 & 40 \\
\hline 50 & 50 & 50 \\
\hline
\end{tabular}

$\mathrm{kWe}$

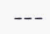

$$
\text { nec }
$$

\begin{tabular}{|c|c|c|}
\cline { 2 - 3 } \multicolumn{1}{c|}{5,316} & 5,316 & 5,316 \\
\hline 0.769 & 0.769 & 0.741 \\
\hline $1,019.8$ & $1,019.8$ & 0 \\
\hline
\end{tabular}

$\mathrm{N}$

1.1378
Reference Mission 16c

\begin{tabular}{|c|c|}
\multicolumn{1}{c|}{ SEPTOP } & Copernicus \\
\hline 1AUx90deg & 1AUx90deg \\
\hline $10 / 1 / 1999$ & $10 / 1 / 1999$ \\
\hline $\begin{array}{c}\text { Delta 7925- } \\
9.5 \text { (LV \#3) }\end{array}$ & -- \\
\cline { 2 - 2 }
\end{tabular}

$4.577-1.226$

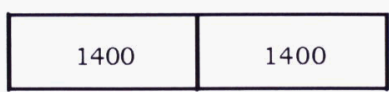

$719.2 \quad 1123.59$

$338.95 \quad 521.96$

\begin{tabular}{|c|c|}
\hline 1324.5 & 1400 \\
\hline "SEPS" model & $1 / \mathrm{R}^{2}$ \\
\hline 7.782 & 7 \\
\hline
\end{tabular}

Electric Engine G

(4 Engines)

$3,337-2,918 \quad 6,455$

$0.58-0.5 \quad 1$

0

$0.2759 \quad 0.2212$

\section{VARITOP RESULTS}

The objective of this mission was to maximize final mass. For this case specific impulse and flight time were variable, and their optimized values resulted in a final mass of $1,121.52$ $\mathrm{kg}$.

\section{MALTO RESULTS}

The MALTO analysis was performed using the simple $1 / R^{2}$ solar array model (where $R$ is the radius from the Sun), and a constrained specific impulse and flight time matching the optimized VARITOP values. Here we can see the affects of changing the solar array model. With all else being the same, MALTO has 24 fewer days spent thrusting. This is explained by referring back to Fig. 2. For distances less than 1 AU from the Sun the $1 / \mathrm{R}^{2}$ solar array model yields higher performance than the "high" array model. The decreased performance of the high model is due to the increased temperatures in the inner solar system. The higher performing $1 / \mathrm{R}^{2}$ model results in a higher final mass of $1138.21 \mathrm{~kg}$ as opposed to VARITOP's $1121.52 \mathrm{~kg}$.

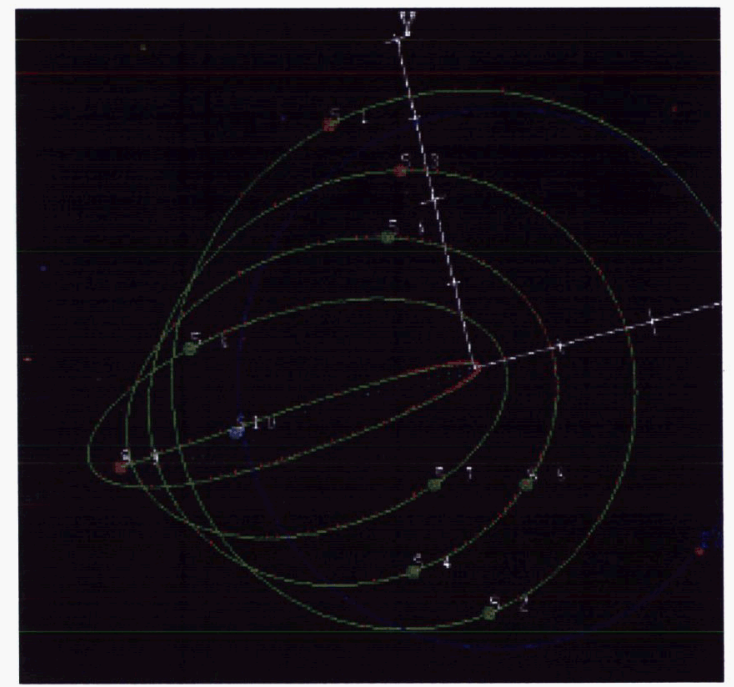

Figure 8. Reference mission $16 \mathrm{c}$ trajectory plot, looking down on the ecliptic plane (Copernicus). 


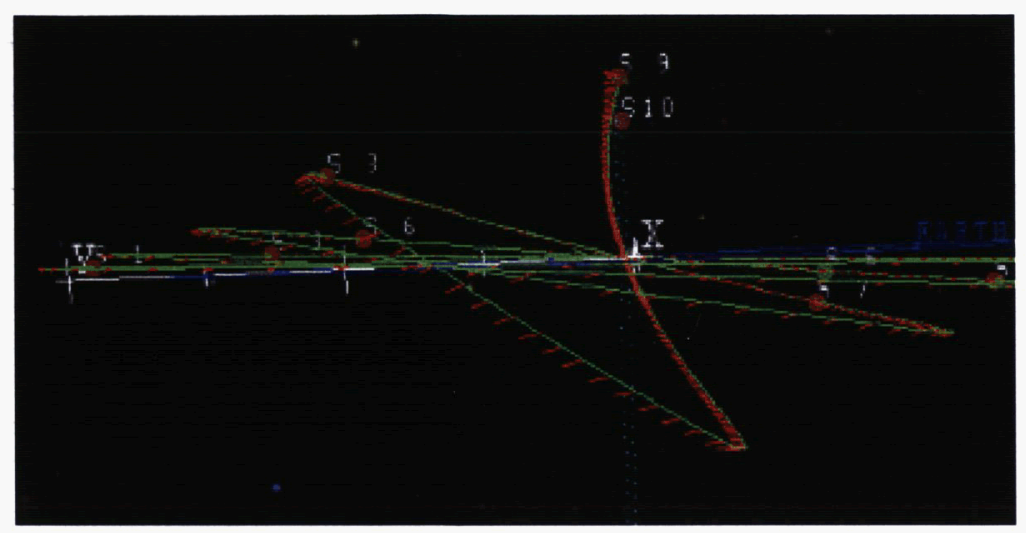

Figure 9. Reference mission 16 trajectory plot, looking from the $-\mathrm{X}$ direction in the plane of the ecliptic (Copernicus).

\section{MYSTIC RESULTS}

The Mystic trajectory is very similar to the MALTO solution. They use the same solar array model, power and specific impulse. The propulsion system efficiency is equivalent. The initial thrust is slightly higher and thrusting time and final mass are different. The values for Mystic's final mass and thrusting time lie between the CHEBYTOP and MALTO values.

4. SEPTOP RESULTS

In this case there are 4 engines and these engines throttle and stage during the mission, so $I_{s p}$ and efficiency are not constant.

\section{COPERNICUS RESULTS}

While the initial mass is not the same as the SEPTOP mission, the ratio of final mass to initial mass is fairly close. The SEPTOP mass ratio is 0.4713 and Copernicus is 0.4645 . Copernicus is not yet able to model the same solar arrays and electric engines as SEPTOP, and since there are a significant number of throttling and staging events this difference in propulsion system modeling most likely makes up most of the differences in performance for this particular mission.

\section{Reference Mission 17: 5-years to Jupiter/Europa Orbiter}

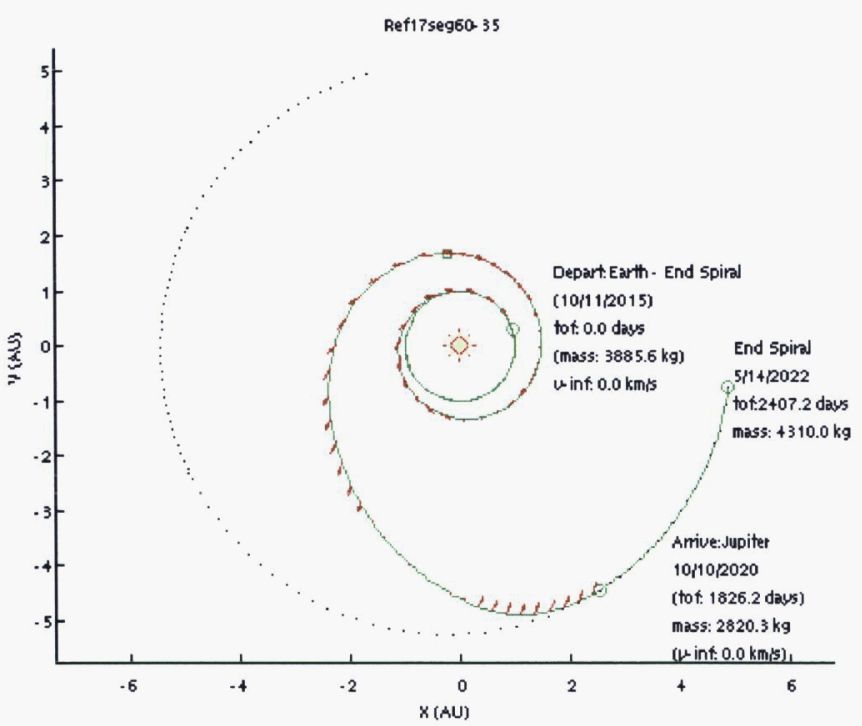

A A nuclear electric powered mission to Jupiter was of great interest to the NASA community just a few years ago. Reference mission 17 represents a straightforward NEP mission to orbit Jupiter. This is the first reference mission in this paper to demonstrate the planet-centered spiraling capability that all of the tools are equipped with. The heliocentric flight time is a constant 5 years for each of the cases, but spiral times for escaping Earth and capturing into an orbit around Jupiter can vary. Figure 10 shows the trajectory path and thrust direction from the MALTO, and the results from each of the tools tested are given in Table 4.

Figure 10. Reference mission 17 trajectory plot (MALTO). 
Table 4. Reference mission 17 results.

\begin{tabular}{|c|c|c|c|c|c|c|c|}
\hline Parameter & Units & CHEBYTOP & VARITOP & MALTO & Copernicus & Mystic & OTIS \\
\hline $\begin{array}{c}\text { Heliocentric } \\
\text { Departure Date }\end{array}$ & $\mathrm{n} / \mathrm{a}$ & $10 / 11 / 2015$ & $10 / 11 / 2015$ & $12 / 11 / 2017$ & $10 / 11 / 2015$ & $10 / 15 / 2015$ & $10 / 11 / 2015$ \\
\hline $\begin{array}{c}\text { Escape Spiral } \\
\text { Time }\end{array}$ & days & 599 & 581 & 595.28 & 620 & 653.48 & --- \\
\hline $\begin{array}{c}\text { Heliocentric Flight } \\
\text { Time }\end{array}$ & days & 1826.25 & 1826.25 & 1826.2 & 1826.25 & 1983.84 & 1826.25 \\
\hline $\begin{array}{c}\text { Capture Spiral } \\
\text { Time }\end{array}$ & days & 586.81 & 575.76 & 600.62 & 776 & 500 & --- \\
\hline $\begin{array}{l}\text { Initial Mass in } \\
\text { Earth Orbit }\end{array}$ & $\mathrm{kg}$ & 4310 & 4310 & 4310 & 4310 & 4310 & --- \\
\hline Mass at Earth SOI & $\mathrm{kg}$ & 3885.6 & 3885.96 & 3885.6 & 3870.7 & 3848.01 & 3885.6 \\
\hline $\begin{array}{c}\text { Mass at Jupiter } \\
\text { SOI }\end{array}$ & $\mathrm{kg}$ & 2846.3 & 2880 & 2921.3 & 2824 & 3064.5 & 2822.3 \\
\hline $\begin{array}{c}\text { Mass in Jupiter } \\
\text { Orbit }\end{array}$ & $\mathrm{kg}$ & 2430.5 & 2460.5 & 2493 & 2274 & 2429.8 & --- \\
\hline $\begin{array}{l}\text { Heliocentric } \\
\text { Thrusting Time }\end{array}$ & days & 1466.75 & 1377.45 & 1352.74 & 1477 & 1163.50 & 1500.7 \\
\hline Specific Impulse & $\sec$ & 6400 & 6400 & 6400 & 6400 & 6400 & 6400 \\
\hline Input Power & $\mathrm{kWe}$ & 24.85 & 25.59 & 25 & 24.85 & 24.85 & 24.85 \\
\hline Efficiency & n.d. & 0.65 & 0.65 & 0.65 & 0.65 & 0.65 & 0.65 \\
\hline Thrust & $\mathrm{N}$ & 0.5147 & 0.5300 & 0.5178 & 0.5147 & 0.5147 & 0.5147 \\
\hline
\end{tabular}

\section{CHEBYTOP RESULTS}

This mission was first modeled in CHEBYTOP. The departure date was manually optimized and then fixed to the optimal value. The heliocentric flight time, initial mass, $I_{s p}$ and efficiency were fixed, but the power was optimized. To maximize payload mass, CHEBYTOP selects an optimal initial power level based on the $I_{s p}$ and propulsion system specific mass, $m_{p s} / P_{0}$, where $m_{p s}$ is defined as follows:

That portion of the initial mass defined as propulsion system mass, $m_{p s}$, includes both the power, $m_{w}$, and the thrust, $m_{t s}$, subsystems, not including propellant tankage but including all internal structure, mechanisms, cabling, thermal control, and so forth. ${ }^{27}$

The propulsion system specific mass for this mission is $50 \mathrm{~kg} / \mathrm{kW}$. The initial power that results in the highest payload delivery is $24.85 \mathrm{~kW}$.

2. VARITOP RESULTS

There are no discrepancies in the inputs to VARITOP. The values and constraints are identical to the CHEBYTOP case; however there is a $30 \mathrm{~kg}(1.2 \%)$ difference in final mass in orbit of Jupiter. VARITOP was able to find a better solution by raising the input power to $25.59 \mathrm{~kW}$.

3. MALTO RESULTS

MALTO was allowed to optimize the departure date, and selected a date 2 synodic periods away from the other cases. This prevents a rigorous comparison since the Earth and Jupiter are not aligned exactly the same, but the final mass is only $33 \mathrm{~kg}$ different from VARITOP (63 $\mathrm{kg}$ more than CHEBYTOP).

\section{COPERNICUS RESULTS}

The final mass is quite a bit lower than for the low and medium fidelity tools. This is most likely due to the differences in escape and capture spiral modeling. CHEBYTOP, VARITOP, and MALTO use a similar approximation for these planet centered spirals, and so it is expected that they would give similar analyses of these mission phases. Mystic and Copernicus both use high fidelity numerical integration for these phases and should be more accurate. However, since Mystic uses different optimization variables, a direct comparison is not possible. 


\section{MYSTIC RESULTS}

This Mystic mission constrains the Earth orbit departure date and optimizes the total flight time. This results in $218 \mathrm{~kg}$ more mass delivered to Jupiter's SOI. However, after the Jupiter capture spiral the final mass is less than 1 $\mathrm{kg}$ different from the CHEBYTOP prediction. The total thrusting time for this case was 2,714 days, which is about 61 days longer than CHEBYTOP. This thrusting time does not match the sum of the thrusting times in Table 4, but the error in the table is likely due to the difficulty in separating out dis tinct mission phases because Mystic optimizes the complete and fully integrated trajectory.

6. OTIS RESULTS

OTIS predicts a mass at Jupiter SOI of $24 \mathrm{~kg}$ lower than CHEBYTOP, and the thrusting time is 33 days longer, however the Copernicus case seems to agree with OTIS with only $2 \mathrm{~kg}$ difference in mass and 23 days difference in thrusting time. With the high fidelity tools OTIS and Copernicus in agreement, it seems that the CHEBYTOP prediction may be off by $0.8 \%$. Escape and capture spirals are not included in this case because OTIS uses the same approximations for these mission phases as is in CHEBYT OP and VARITOP.

\section{E. Reference Mission 26: 12-years to Pluto Tour}

Reference mission 26 has only been analyzed by one tool so far so we can't make any comparisons, but it is included in this paper to demonstrate the range of mission analysis capabilities that are available with the LTTT tools. The heliocentric portion of this mission is modeled in reference mission 21 (available on the LTTT website). Figs. 11 and 12 show the Pluto vicinity trajectory in Charon-centered and Pluto-centered reference frames respectively. Data on this mission is given in Table 5.

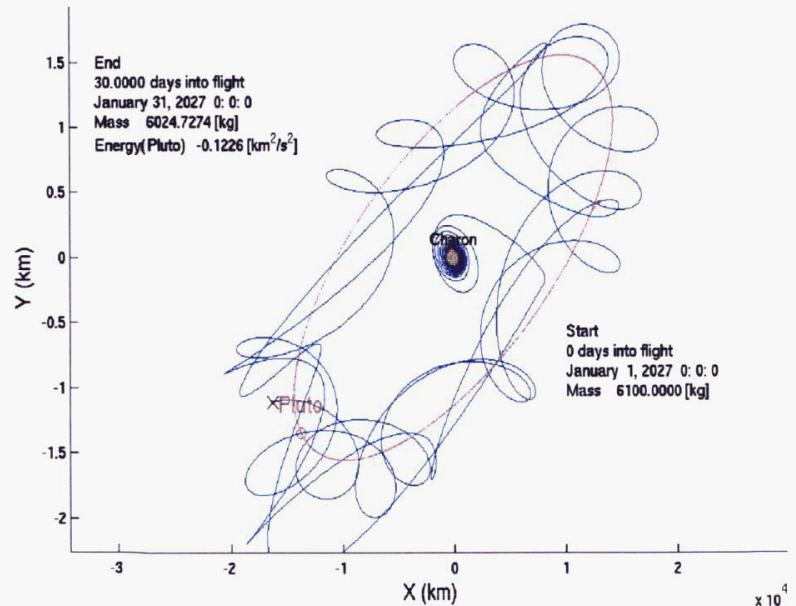

Figure 11. Reference mission 26 Charon-centered trajectory plot (Mystic).

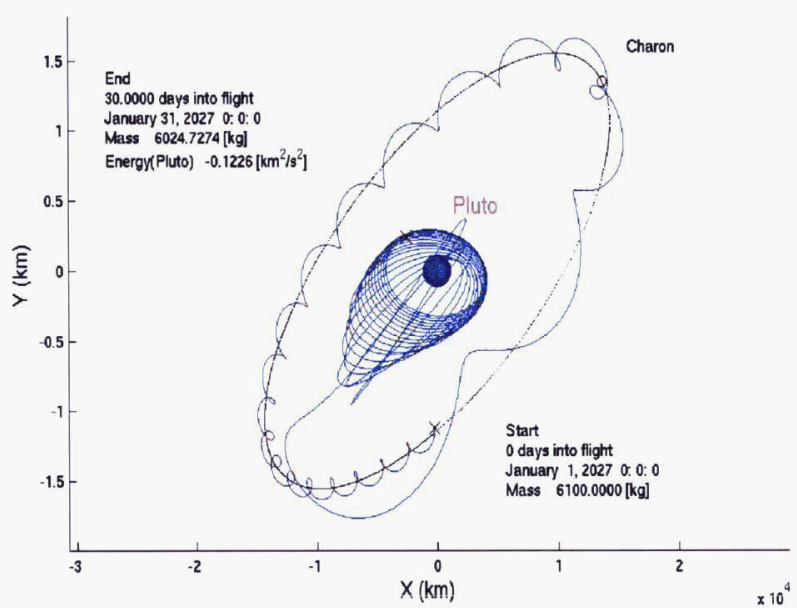

Figure 12. Reference mission 26 Pluto-centered trajectory plot (Mystic).

\section{F. Reference Mission 28a \& 28c: Earth - Moon low-thrust \& Earth - Lunar L1 Halo Orbit}

Reference missions 28 and 28c demonstrate the capability of Copernicus and Mystic to operate in the Earth vicinity with multiple gravity fields. SNAP and OTIS have this capability as well, but have not yet completed this reference mission. Figures 13 and 14 illustrate reference missions 28 and $28 \mathrm{c}$ respectively. Data for reference mission 28 is given in Table 6.

\section{COPERNICUS RESULTS}

The Copernicus case reaches the Moon in a little over 2 months. There may be a discrepancy in the final target orbit. This is currently under investigation, and may be the cause of the large difference in flight time.

2. MYSTIC RESULTS

Mystic's mission captures into an orbit of the Moon after 81 days, and arrives in the final desired orbit of the Moon in a total of 186 days. 
Table5. Reference mission 26 results .

\begin{tabular}{|c|c|c|}
\hline Parameter & Units & Mystic \\
\hline Departure Date & $\mathrm{n} / \mathrm{a}$ & $1 / 1 / 2015$ \\
\hline $\begin{array}{c}\text { Helocentric Flight } \\
\text { Time }\end{array}$ & days & 4,383 \\
\hline Initial Mass & $\mathrm{kg}$ & 15,347 \\
\hline $\begin{array}{c}\text { Arrive Charon Polar } \\
\text { Orbit }\end{array}$ & $\mathrm{n} / \mathrm{a}$ & $1 / 1 / 2027$ \\
\hline Alt above Charon & $\mathrm{km}$ & 100 \\
\hline $\begin{array}{c}\text { Mass Orbiting } \\
\text { Charon }\end{array}$ & $\mathrm{kg}$ & 6,100 \\
\hline $\begin{array}{c}\text { Arrive Low Pluto } \\
\text { Orbit }\end{array}$ & $\mathrm{n} / \mathrm{a}$ & $2 / 14 / 2027$ \\
\hline Alt above Pluto & $\mathrm{km}$ & 100 \\
\hline Tour Flight Time & days & 44 \\
\hline Final Mass & $\mathrm{kg}$ & 5,988 \\
\hline Specific Impulse & sec & 6100 \\
\hline Input Power (NEP) & $\mathrm{kWe}$ & 84.053 \\
\hline Efficiency & n.d. & 0.65 \\
\hline
\end{tabular}

Table 6. Reference mission 28a results.

\begin{tabular}{|c|c|c|c|}
\hline Parameter & Units & Copernicus & Mystic \\
\hline Departure Date & $\mathrm{n} / \mathrm{a}$ & $4 / 30 / 2003$ & $5 / 20 / 2003$ \\
\hline $\begin{array}{c}\text { Lunar Capture } \\
\text { Date }\end{array}$ & $\mathrm{n} / \mathrm{a}$ & $7 / 6 / 2003$ & $8 / 9 / 2003$ \\
\hline Flight Time & days & 67.25 & 186 \\
\hline Initial Mass & $\mathrm{kg}$ & 2500 & 2500 \\
\hline $\begin{array}{c}\text { Lunar Capture } \\
\text { Mass }\end{array}$ & $\mathrm{kg}$ & --- & 2473.7 \\
\hline Final Mass & $\mathrm{kg}$ & 2463.6 & 2418.3 \\
\hline Solar Array & & NEP & NEP \\
\hline Input Power & $\mathrm{kWe}$ & 14 & 20 \\
\hline Specific Impulse & $\mathrm{sec}$ & 6000 & 6000 \\
\hline Efficiency & n.d. & 1 & 0.7 \\
\hline Initial Thrust & $\mathrm{N}$ & 0.4759 & 0.4643 \\
\hline
\end{tabular}

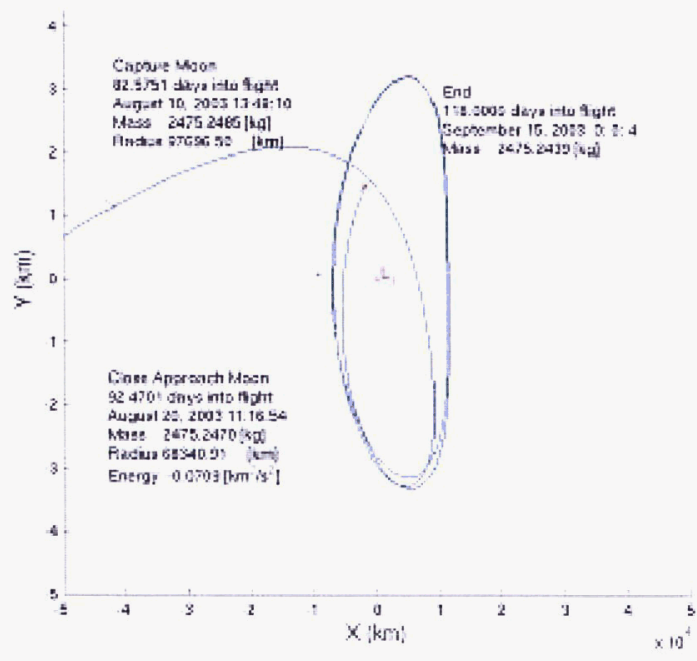

Figure 14: Reference Mission 28c Trajectory Plot (Mystic). 


\section{Future Work}

A NASA Technical Memorandum, or TM, will be drafted over the next few months to fully document reference missions and beta testing results. Sixty five reference mission cases are complete for the 5 LTTT tools. This represents about half of the cases that can be modeled with these tools. Work will continue to complete the remaining cases as time and resources allow. Particular attention will be given to OTIS and SNAP cases, as they are the least represented of the suite of 5 LTTT tools.

\section{Conclusions}

One of the goals of this paper was to determine performance prediction differences between tools of different fidelity levels. It is apparent, especially in reference mission 1, that low, medium, and high fidelity tools can arrive at very similar answers. What high fidelity buys you is not always higher accuracy, but the ability to model more complex missions. One can see from the data presented in this paper that lower fidelity tools can predict masses within $1-2 \%$ of high fidelity tools when used for the missions they were designed for, and lower fidelity tools often have benefits over the high fidelity tools. They can provide quicker execution time, rapid trade study analysis, and they are often much easier to learn. Mission requirements and study objectives should be key drivers in determining the most appropriate tool for the job.

Comparison of the high fidelity tools is a challenge at this time, because in all of the missions discussed in this paper differences in propulsion system modeling, and dissimilar constraints introduce variation into the comparison. However, Copernicus, Mystic, and OTIS compare well with each other in reference mission 1 and Copernicus and OTIS compare well in reference mission 17. Final masses are within $1 \%$ of each other. As beta testing continues, discrepancies in constraints will be corrected and more cases will be available to compare all of the LTTT high fidelity tools: Mystic, Copernicus, OTIS, and eventually SNAP.

Without going into a rigorous comparison of ephemeris data, numerical integration, and optimization schemes, the results in this paper should give the reader a general sense of how well the tools can compare to each other. In many cases it may be possible to improve the comparisons by adjusting constraints and models to more closely match between the tools. And, while matching between tools does not validate the results in a strict sense (only flight demonstration or flight qualified software can do that), it should give the reader and future users confidence in the capabilities of these tools. The distribution and independence of the development teams make it unlikely that all of the tools could be wrong in the same way. Eventually, Mystic may complete the flight software validation process, and can then be used to validate the other tools.

\section{Acknowledgments}

The results presented in this paper were generated by the authors and by Try Lam, Dr. Cesar Ocampo, Dr. Greg Whiffen, Steve Williams, John Riehl, and Leon Gefert. The authors would like to give special recognition to Try Lam of JPL for single -handedly generating almost half of the beta test cases that are currently available on the LTTT website, and for providing critical and timely support during the writing of this paper.

The authors would like to thank all of the developers and beta testers at the Jet Propulsion Laboratory, Johnson Space Center, and the University of Texas at Austin, and at Glenn Research Center and The Boeing Company for their patience and constant assistance. Special thanks go out to Try Lam, Dr. Jon Sims, Dr. Cesar Ocampo, Theresa Kowalkowski, Ed Rinderle, Matt Vavrina, Ravi Mathur, Fady Morcos, Dr. Greg Whiffen, and John Riehl, for exceptional support to the beta testing process.

The authors would also like to thank the In-Space Propulsion Office for fighting budget cuts and seeing this tremendous project through to fruition.

\section{References}

\footnotetext{
${ }^{1}$ Kos, L., "Overview of the Development of a Suite of Low-Thrust Trajectory Analysis Tools," AIAA/AAS Astrodynamics Specialist Conference, AIAA-2006-6743, Keystone, Colorado, August 21-24, 2006. CHEBYTOP References

${ }^{2}$ Hahn, D. W., Johnson, F.T., and Itzen, B. F., "Final Report for CHEBYCHEV Optimization Program (CHEBYTOP)," The Boeing Company, Report No. D2-121308-1, NASA-CR-73359 (or 69N34537), July 1969.

${ }^{3}$ Hahn, D. W. and Johnson, F.T., "Final Report for CHEBYCHEV Optimization Program (CHEBYTOP II)," The Boeing Company, Report No. D180-12916-1, NASA-CR-114354 (or 71N30678), June 1971.

"Johnson, F.T., "Final Report for the Improvement of the [illegible] Digital Computer Program CHEBYTOP II," The Boeing Company, Report No. D180-15371-1, NASA-CR-114505 (or 73x10397), April 1973.
} 
${ }^{5}$ Johnson, F. T., "Approximate Finite-Thrust Trajectory Optimization," AIAA Journal, Vol. 7, No. 6, June 1969, pp. 993-997 VARITOP/SEPTOP/Sail references

${ }^{6}$ Sauer, Carl G., Jr., "A Preliminary User's Guide to VARITOP, SAIL, and SEPTOP General Purpose Low-Thrust Trajectory Optimization Programs," Jet Propulsion Laboratory, Pasadena, CA, February 2002.

MALTO References

${ }^{7}$ Sims, Jon A., Finlayson, Paul A., Vavrina, Matthew A., and Kowalkowski, Theresa D., "Implementation of a Low-Thrust Trajectory Optimization Algorithm for Preliminary Design," AIAA/AAS Astrodynamics Specialist Conference, AIAA-20066746, Keystone, Colorado, August 21-24, 2006.

${ }^{8}$ Kowalkowski, T., Rinderle, E., "MALTO Users' Manual, Version 4.2," Draft Version 0.8, Jet Propulsion Laboratory, Pasadena, CA, November 9, 2005

${ }^{9}$ Sims, Jon A., and Flanagan, Steve N., "Preliminary Design of Low-Thrust Interplanetary Missions," AAS/AIAA Astrodynamics Specialist Conference, AAS 99-338, Girdwood, Alaska, August 16-18, 1999.

${ }^{10}$ McConaghy, T. T., Debban T. J., Petropoulos, A. E., and Longuski, J. M., "Design and Optimization of Low-Thrust Trajectories with Gravity Assists," Journal of Spacecraft and Rockets, Vol. 40, No. 3, 2003, pp.380-387.

${ }^{11}$ Gill, Philip E., "User's Guide for SNOPT Version 7: A FORTRAN Package for Large-Scale Nonlinear Programming." University of California, San Diego, December 4, 2004.

Copernicus References

${ }^{12}$ Condon, Gerald L., Ocampo, C., Senent, J., "The Development and Use of COPERNICUS: A Comprehensive Trajectory Design and Optimization System," AIAA/AAS Astrodynamics Specialist Conference, AIAA-2006-6740, Keystone, Colorado, August 21-24, 2006.

${ }^{13}$ Ocampo, C., "An Architecture for a Generalized Trajectory Design and Optimization System," available at URL: http://www.ieec.fcr.es/libpoint/abstracts/ocampo.pdf [cited 31 July 2006] .

${ }^{14}$ Ocampo, C., "Finite Burn Maneuver Modeling for a Generalized Spacecraft Trajectory Design and Optimization System," Annals of the New York Academy of Sciences [online journal], Vol. 1017, 210-233, URL: http://www.annalsnyas.org/cgi/content/abstract/1017/1/210 [cited 31 July 2006].

Mystic References

${ }^{15}$ Whiffen, Gregory J., "Mystic: Implementation of the Static Dynamic Optimal Control Algorithm for High-Fidelity, LowThrust Trajectory Design," AIAA/AAS Astrodynamics Specialist Conference AIAA-2006-6741, Keystone, Colorado, August 21-24, 2006.

${ }^{16}$ Whiffen, G. J., "MYSTIC, Low-Thrust Trajectory Optimization Toolset, User's Manual for Version 9.0.1," Outer Planets Mission Design Group, Jet Propulsion Laboratory, Pasadena, California, March 15, 2006.

${ }^{17}$ Whiffen, G. J., and Sims, J. A., "Application of the SDC Optimal Control Algorithm To Low-Thrust Escape and Capture Trajectory Optimization," Paper AAS 02-208, AAS/AIAA Space Flight Mechanics Conference, San Antonio, Texas, USA, January 27-30, 2002.

${ }^{18}$ Whiffen, G. A., and Sims, J. A., "Application of a Novel Optimal Control Algorithm to Low-Thrust Trajectory Optimization," AAS/AIAA Space Flight Mechanics Conference, AAS Paper 01-209, Santa Barbara, CA, February 11-15, 2001.

${ }^{19}$ Whiffen, G. J., "Static/Dynamic Control for Optimizing a Useful Objective," United States Patent No.: 6,496,741, Issued December 17, 2002, Filed March 25, 1999.

OTIS References

${ }^{20}$ John P. Riehl, Paris, Steve, Sjauw, W., "Comparison of High Order Collocation, Pseudo-spectral, and Runge-Kutta Methods of Numerical Trajectory Optimization in Aerospace Problems," AIAA/AAS Astrodynamics Specialist Conference AIAA-2006-6033, Keystone, Colorado, August 21-24, 2006.

${ }^{21}$ Paris, S. W., Riehl, J. P., and Sjauw, W., "Enhanced Procedures for Direct Trajectory Optimization Using Nonlinear Programming and Implicit Integration," AIAA/AAS Astrodynamics Specialist Conference AIAA-2006-6309, Keystone, Colorado, August 21-24, 2006.

22“"Optimal Trajectories by Implicit Simulation, OTIS, Volume I - Formulation Manual," March 2006.

${ }^{23}$ Kraft, Dieter, "A Software Package for Sequential Quadratic Programming". DFVLR-FB 88-28, Koln, Germany

SNAP References

${ }^{24}$ Martini, M., "S.N.A.P. 2.3 User's Manual, SPACECRAFT N-BODY ANALYSIS PROGRAM," December, 12, 2005. Other References

${ }^{25}$ Ellis, Jordan, Lisano, Michael, Wolff, Peter, Evans, James, Bladt, Jeff, Scheeres, Dan, Rios-Reyes, Leonel, Lawrence, Dale, "A Solar Sail Integrated Simulation Toolkit," AAS/AIAA Space Flight Mechanics Conference, AAS04-283, Maui, Hawaii, February 2004.

${ }^{26}$ Heaton, Andrew F., "Solar Sail GN\&C Model Comparisons," AIAA Guidance, Navigation, and Control Conference and Exhibit, AlAA 2004-4892, Providence, Rhode Island, August 16-19, 2004

${ }^{27}$ Electric Propulsion Mission Analysis Terminology \& Nomenclature," NASA SP-210, 1969 